\title{
Protective effects of three remote ischemic conditioning procedures against renal ischemic/reperfusion injury in rat kidneys: a comparative study
}

\author{
H. Jiang $\cdot$ R. Chen $\cdot$ S. Xue $\cdot$ H. Zhu $\cdot$ \\ Xiaolei Sun · Xiaoqing Sun
}

Received: 10 April 2014/Accepted: 1 November 2014/Published online: 15 November 2014

(C) The Author(s) 2014. This article is published with open access at Springerlink.com

\begin{abstract}
Background Remote ischemic perconditioning (RIPerC), remote ischemic postconditioning (RIPostC), and remote ischemic perconditioning + postconditioning (RIPerC + RIPostC) protect against renal ischemia reperfusion injury (IRI). However, the most beneficial approach among these is not known.

Aims To compare the protective effects and study the mechanisms of three different remote ischemic conditioning in preventing IRI in the rat kidney.

Methods Fifty healthy adult male Sprague-Dawley rats were randomly assigned to five groups: sham, IRI, RIPerC, RIPostC, and RIPerC + RIPostC. Right nephrectomy was performed initially in all rats. IRI was induced by occluding the left renal artery for $60 \mathrm{~min}$, followed by reperfusion for 24 h. RIPerC, RIPostC, and RIPerC + RIPostC were induced with 5-min ischemia/reperfusion (I/R) cycles using a tourniquet on the right hind limb.

Results The IRI group showed significant serologic evidence of renal injury compared to the sham group $(P<0.05)$. The RIPerC, RIPostC, and RIperC + RIpostC groups displayed significantly lower levels of renal
\end{abstract}

H. Jiang $\cdot$ R. Chen $(\varangle) \cdot$ S. Xue $\cdot$ H. Zhu $\cdot$ Xiaolei Sun The Department of Urology, The Affiliated Hospital of Xuzhou Medical College, 99 Huaihai West Road,

Xuzhou 221000, Jiangsu, China

e-mail: Renfu_chen@aliyun.com

H. Jiang

e-mail: Jhb870514@126.com

\section{Xiaoqing Sun $(\bowtie)$}

The Urinary Laboratory, The Affiliated Hospital of Xuzhou

Medical College, 99 Huaihai West Road,

Xuzhou 221000, Jiangsu, China

e-mail: Sunheyao@126.com dysfunction than the IRI group $(P<0.05)$. Superoxide dismutase (SOD) levels were significantly lower in the IRI group than in the sham group $(P=0.003)$, but were significantly less depressed in the RIPerC, RIPostC, and RIperC + RIpostC groups $(P<0.05)$. The IRI group displayed more severe renal tubular injury than the RIPerC, RIPostC, and RIPerC + RIPostC groups $(P<0.05)$.

Conclusion All three remote ischemic conditioning showed similar therapeutic potential for preventing renal IRI. The RIPerC + RIPostC protocol did not show an additive effect from the combination of preconditioning and postconditioning. The protective mechanism may be due to the stimulation of endogenous antioxidant activity by transient limb ischemia-reperfusion.

Keywords Remote ischemic perconditioning - Remote ischemic postconditioning $\cdot$ Kidney $\cdot$ Ischemic reperfusion injury

\section{Introduction}

Ischemia-reperfusion injury (IRI) occurs when blood supply to a tissue is temporarily interrupted. When blood flow is restored reperfusion paradoxically induces more severe tissue injury $[1,2]$. Renal warm-IRI occurs in clinical practice and is a consequence of systemic hypoperfusion with subsequent circulatory resuscitation. Local nephritic hypoperfusion after aortic cross-clamping or renal transplantation also causes IRI to the kidney.

Several recent trials have shown that remote ischemic conditioning (RIC) has a powerful protective effect in limiting nephritic IRI [3]. RIC is accomplished with brief nonlethal cycles of ischemia and reperfusion of an arm or leg. These cycles may be applied before (preconditioning), 
during (perconditioning) [4], or after (postconditioning) [5] prolonged ischemia of a distant organ [6, 7]. Some studies have found that RIC is a straightforward, inexpensive, noninvasive, and powerful means of preventing nephritic IRI during surgery or organ transplantation [3, 8, 9].

In previous studies, remote ischemic postconditioning (RIPostC) and perconditioning (RIPerC) have provided practical methods for protecting the kidneys against IRI [3], but their combined effects and mechanism have not been studied in detail.

In the present study, we conducted a randomized trial on rats in which we induced IRI. To augment the protective effect of RIC, RIC was induced through lower limb ischemia, rather than upper limb ischemia, and RIPerC was combined with RIPostC (RIPerC + RIPostC). Renal IRI was assessed by measuring levels of serum creatinine ( $\mathrm{SCr}$ ) and blood urea nitrogen (BUN). We also histologically assessed the degree of renal tubular injury, and measured myeloperoxidase (MPO) activity superoxide dismutase (SOD) activity and malondialdehyde (MDA) content.

\section{Materials and methods}

\section{Animals}

Eighteen-week-old male Sprague-Dawley rats, weighing between 200 and $250 \mathrm{~g}$ (Experimental Animal Center, Xuzhou Medical College, Xuzhou, Jiangsu province, China) were studied. The animal research study protocol was in compliance with the Guide for the Care and Use of Laboratory Animals published by the National Institutes of Health (NIH Pub. No. 85-23, revised 1996) and approved by the Animal Care Committee of the Affiliated Hospital of Xuzhou Medical College, Xuzhou Medical College. All rats were acclimatized with free access to food and water in a $22-27^{\circ} \mathrm{C}$ environment for 2 weeks prior to the experiments.

\section{Experimental design and surgical procedure}

All rats were fasted $12 \mathrm{~h}$ before surgery. Surgical procedures were performed with the rats under sodium pentobarbital anaesthesia (40 mg/kg; I.P.). The RIC stimulus was delivered via tourniquet blockage of blood flow to the right hind limb for cycles of 5-min occlusion followed by 5-min resumption of blood flow. Fifty rats were randomly allocated to each of five experimental groups; three rats were excluded because of anaesthetic or surgical complications. All animals underwent right nephrectomy. In the IRI group $(n=10)$, the left renal artery was occluded for $60 \mathrm{~min}$ with a nontraumatic vascular clip, followed by $24 \mathrm{~h}$ of reperfusion. In the sham group $(n=10)$, all the above surgical procedures were performed, except that IRI was not induced. In the RIPerC group $(n=8)$, four cycles of $5 \mathrm{~min}$ of ischemia followed by $5 \mathrm{~min}$ of reperfusion were performed on the right hind limb during renal ischemia and before renal reperfusion. In the RIPostC group $(n=9)$, four cycles of ischemia/reperfusion of the right lower limb were performed immediately after restoring blood flow to the kidney. In the RIPerC + RIPostC group ( $n=10)$, two cycles of ischemia/reperfusion were performed during renal ischemia before renal reperfusion, and two similar cycles were performed immediately upon restoring blood flow to the kidney (Fig. 1).

Measurement of SCr and BUN concentrations

At the end of the 24-h reperfusion period, plasma samples were collected, and SCr and BUN levels were determined using commercially available colorimetric methods, according to the manufacturer's instructions.

Measurement of MPO, MDA, and SOD in renal tissue

Renal tissue samples were collected and $10 \%$ homogenate samples were prepared. MPO activity, MDA content, and SOD activity were measured in the $10 \%$ homogenates by colorimetric methods using commercially available kits, according to the manufacturer's instructions.

\section{Histology}

In each rat, the left kidney was removed under fully maintained anaesthesia. Animals were sacrificed only after removal of the left kidney. After removal, the kidney was bisected along the non-hilar axis and was fixed in $10 \%$ phosphate-buffered formalin. The tissues were subsequently embedded in paraffin, sectioned, stained with hematoxylin and eosin, and were analyzed. Renal damaged was histologically graded using the established by accepted grading system described by Jablonski et al. [10]. Kidney injury was scored by a single pathologist (X.L.S.) as the percentage of damaged tubules in the corticomedullary junction. Criteria for kidney injury included tubular necrosis, cast formation, loss of the brush border, tubular dilatation and immune cell infiltration. Scoring for each category was as follows: 0 for no change; 1 for $<10 \% ; 2$ for $10-20 \%$; 3 for $21-30 \% ; 4$ for $>30 \%$ area change. Scores for all categories were added for the final injury score.

Calculations and statistics

Graph Pad Prism 5 (Graph Pad Inc., La Jolla, CA) was used to analyze and present data. Differences between groups were analyzed using a paired parametric $t$ test or a one-way 


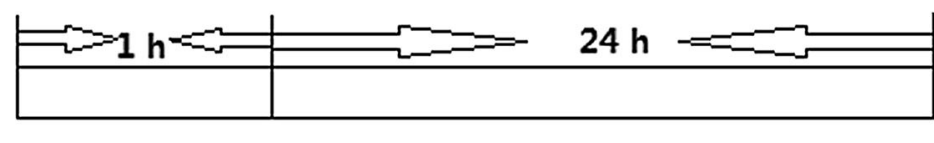

sham

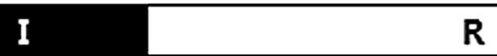

IRI

\begin{tabular}{|c|c|c|}
\hline $\mathbf{I}$ & $\mathbf{R}$ & target organ \\
\hline$\square \mathrm{I}^{-}$ & & remote organ \\
\hline
\end{tabular}

RIPerC

\begin{tabular}{|l|l|l|l|}
\hline $\mathbf{I}$ & \multicolumn{2}{|c|}{ target organ } \\
\hline & $\mathrm{R}$ & \multicolumn{1}{|c|}{ remote organ } \\
\hline
\end{tabular}

RIPostC

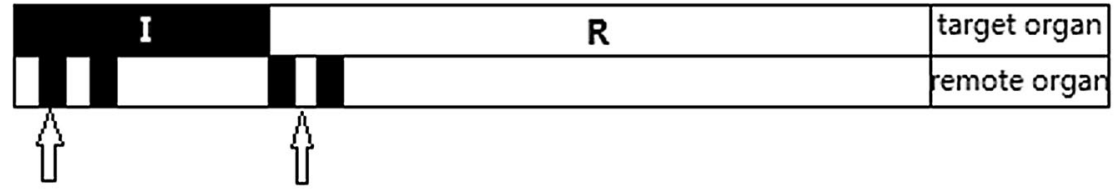

RIPerC+RIPostC

Ischemia $5 \mathrm{~min}$

Reperfusion 5 min

Fig. 1 Experimental protocols and grouping of the animals

A SCr levels among five groups at $24 \mathrm{~h}$ after reperfusion

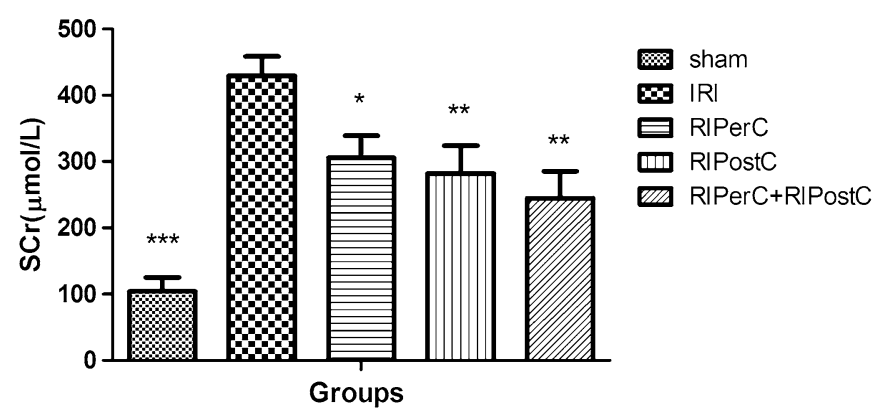

Fig. 2 a and b, respectively demonstrate that $\mathrm{SCr}$ and BUN concentrations in the IRI, RIPerC, RIPostC, and RIPerC + RIPostC groups are significantly higher than those in the sham group. In

ANOVA test. Values are expressed as the mean \pm SEM. A $P$ value $<0.05$ was considered statistically significant.

\section{Results}

Functional assessment

As shown in Fig. 2a, b, the levels of $\mathrm{SCr}$ and BUN in the IRI, RIPerC, RIPostC and RIPerC + RIPostC groups were significantly higher than seen in the sham group $(P<0.001)$. In addition, the IRI group showed higher levels of SCr and BUN than the RIPerC, RIPostC, and RIPerC + RIPostC groups. There was no significant difference in $\mathrm{SCr}$ and BUN levels among the RIPerC,
B BUN levels among five groups at $24 \mathrm{~h}$ after reperfusion

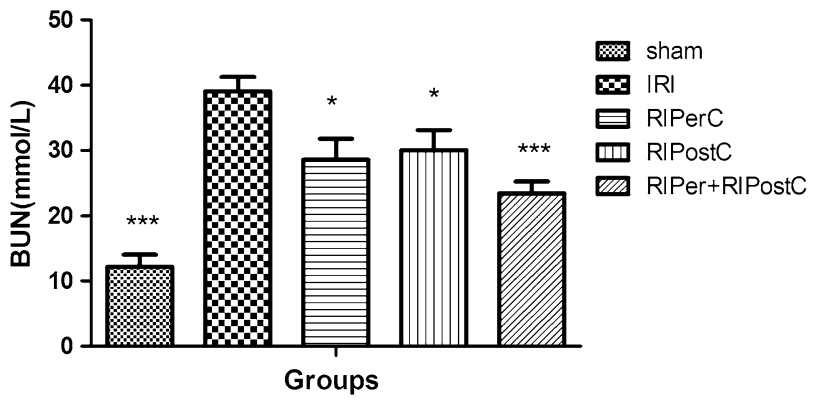

addition, the IRI group shows higher $\mathrm{SCr}$ and BUN concentrations than the RIPerC, RIPostC, and RIPerC + RIPostC groups. (*** $P<0.001$, ** $P<0.01, * P<0.05$ vs. IRI group)

RIPostC, and RIPerC + RIPostC groups at $24 \mathrm{~h}$ after reperfusion $(P>0.05)$. These results indicate that the renal IRI induced by a 60 -min period of ischemia can be limited to some degree using RIC.

SOD activity, MPO activity, and MDA content

MPO activity and MDA content levels were significantly elevated in the IRI group compared to the sham group. All of the RIC groups (RIPerC, RIPostC, and RIPerC + RIPostC) had a less remarkable elevation of MPO activity and MDA content $(P<0.05$, Fig. 3a, c). SOD activity significantly decreased in the IRI group compared to that in the sham group, but did not significantly decrease in the RIC groups $(P<0.05$, Fig. $3 b)$. SOD activity, MPO activity, and MDA 
A $24 \mathrm{~h}$ after reperfusion MPO activity in groups

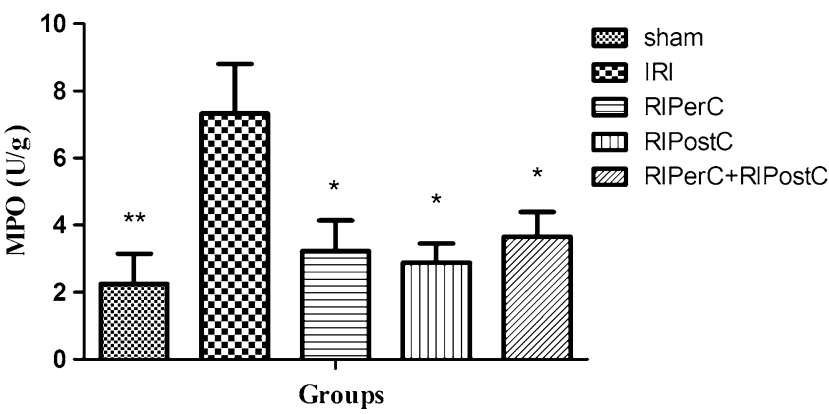

C $24 \mathrm{~h}$ after reperfusion MDA content in groups

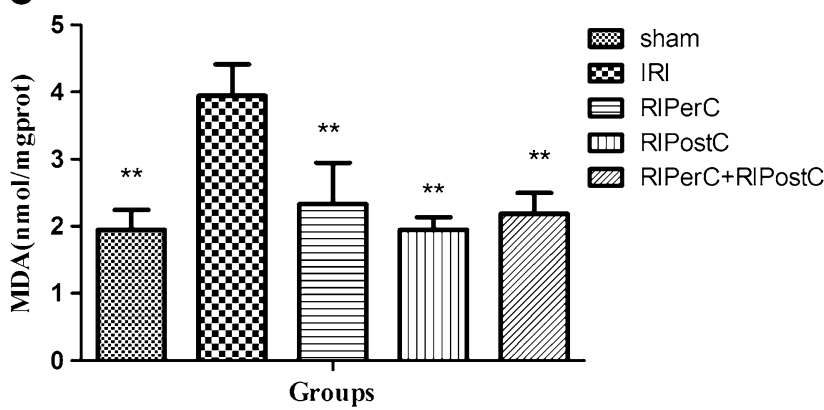

Fig. 3 a and c, respectively, demonstrate that MPO activity and MDA content levels are significantly elevated in the IRI group compared to those in the sham group. All of the RIC groups (RIPerC, RIPostC, and RIPerC + RIPostC) had a lesser elevation of MPO activity and MDA content. c Demonstrates that SOD activity significantly decreased in the IRI group compared to that in the

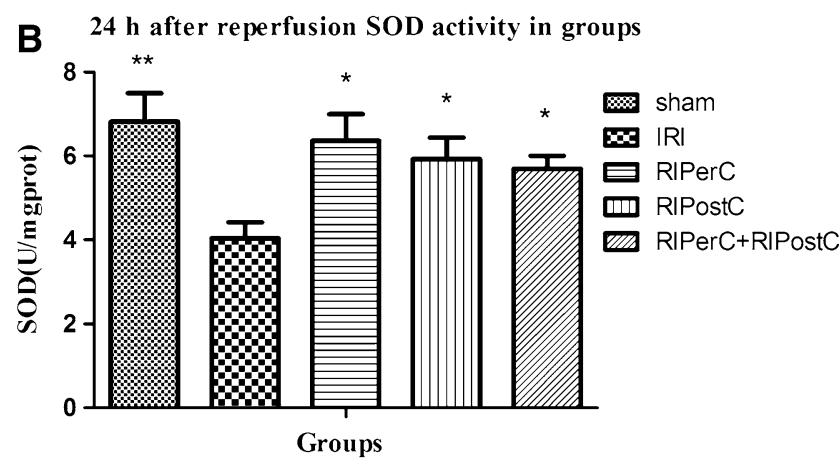

D $24 \mathrm{~h}$ after reperfusion scores of damaged tubules in groups

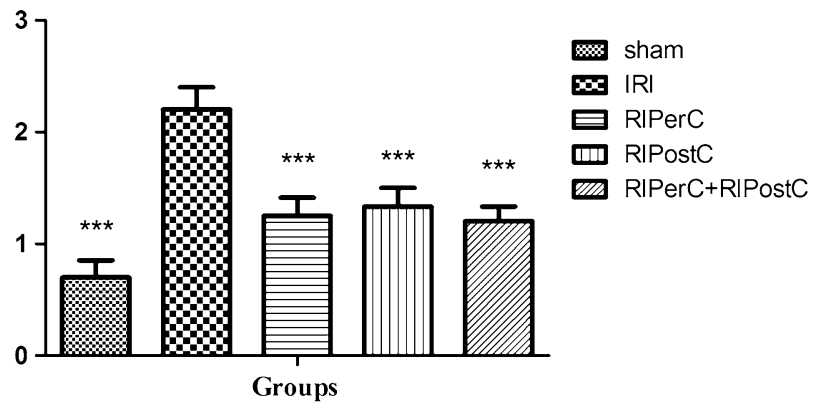

sham group, but did not significantly decrease in the RIC groups (RIPerC, RIPostC, and RIPerC + RIPostC groups). d Shows that the tubular damage score significantly decreased in the RIC groups (RIPerC, RIPostC, and RIPerC + RIPostC groups) compared to that the IRI group. $(* * * P<0.001, * * P<0.01, * P<0.05$ vs. IRI group)
Table 1 Renal tubular damage scores in the five groups

\begin{tabular}{llllll}
\hline Groups & \multicolumn{4}{l}{ Degree of damage } & \\
\cline { 2 - 6 } & $\begin{array}{l}\text { No } \\
\text { change }(n)\end{array}$ & $\begin{array}{l}\text { Minimal } \\
(<10 \%)(n)\end{array}$ & $\begin{array}{l}\text { Mild } \\
(11-20 \%)\end{array}$ & $\begin{array}{l}\text { Moderate } \\
(21-30 \%)(n)\end{array}$ & $\begin{array}{l}\text { Severe } \\
(>30 \%)(n)\end{array}$ \\
\hline Sham $(n=10)$ & 3 & 7 & 0 & 0 & 0 \\
IRI $(n=10)$ & 0 & 1 & 6 & 3 & 0 \\
RIPerC $(n=8)$ & 0 & 6 & 2 & 0 & 0 \\
RIPostC $(n=9)$ & 0 & 6 & 3 & 0 & 0 \\
RIPerC + RIPostC & 0 & 8 & 2 & 0 & 0 \\
$(n=10)$ & & & & &
\end{tabular}

content were not significantly different between the RIPerC, RIPostC and RIPerC + RIPostC groups. ( $P>0.05$, Fig. 3).

\section{Histological assessment}

According to the well-known grading system established by Jablonski et al. the extent of renal tubular damage in the study groups is described in detail in Table 1. The RIPerC, RIPostC, and RIPerC + RIPostC groups showed ischemic tubulointerstitial abnormalities, that were clearly less prominent than those seen in the IRI group, which displayed moderate-to-severe ischemic-characteristic tubulointerstitial lesions $(P<0.05$, Figs. 3d, 4). A significant difference was not seen among the RIPerC, RIPostC, and RIPerC + RIPostC groups at $24 \mathrm{~h}$ after kidney reperfusion.

\section{Discussion}

The present study shows that RIPerC + RIPostC was as effective as RIPerC or RIPostC in decreasing renal reperfusion injury in a rat model of IRI. However, the RIPerC + RIPostC combination did not result in superior outcomes to either RIPerC or RIPostC alone.

The renal IRI protective effect of RIPerC and RIPostC was first reported by Kadkhodaee et al. [3] in 2011. In a rat 


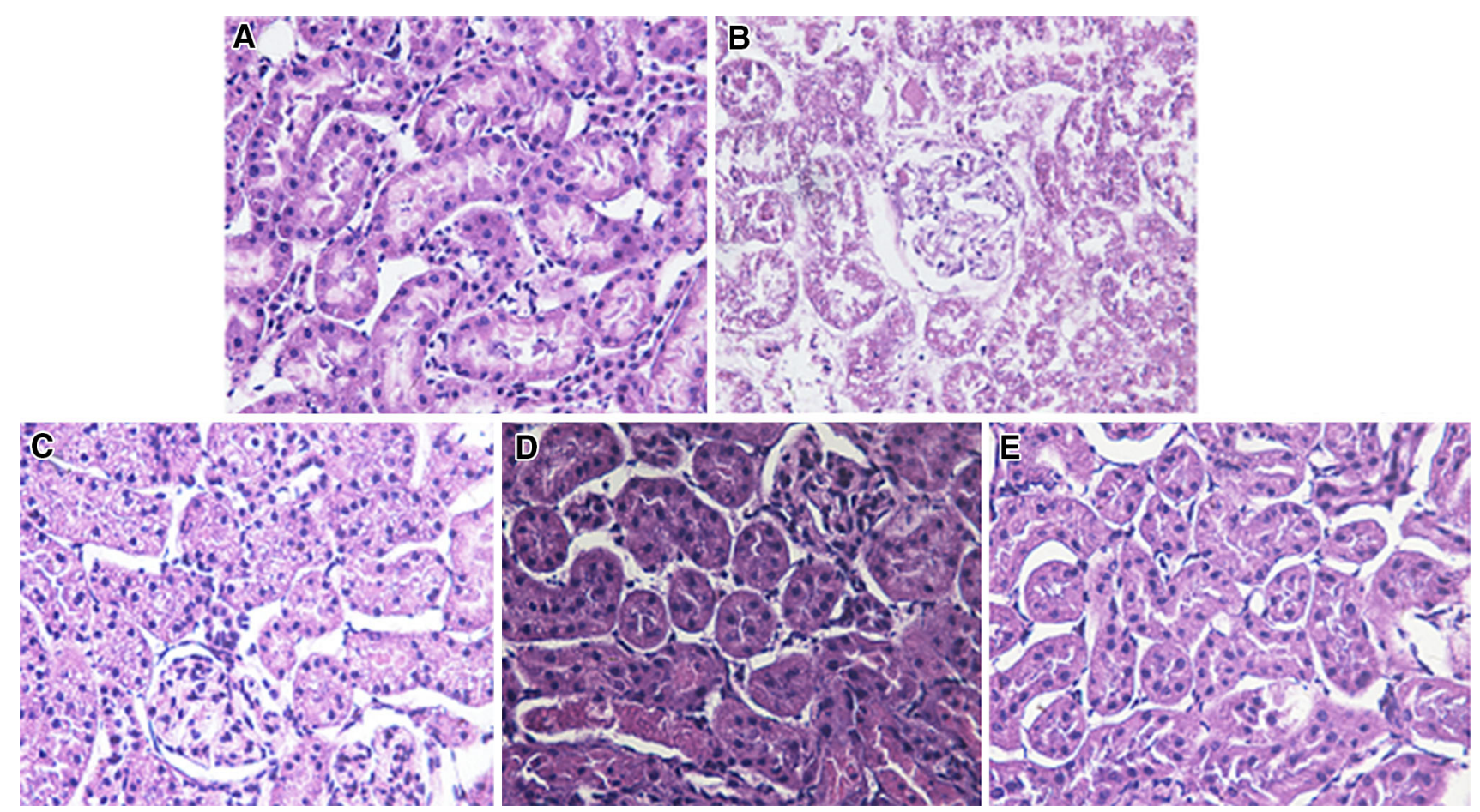

Fig. 4 Tissue sections stained with hematoxylin and eosin (H\&E) $\times 40$. a Sham operation (sham group): no abnormalities; $\mathbf{b}$ untreated IRI (IRI group): moderate-to-severe tubular cell necrosis, tubular dilation, intratubular cell detachment, interstitial oedema, and

model of nephritic IRI, four episodes of 5-min ischemia followed by 5-min reperfusion of the left femoral artery were applied during renal ischemia before reperfusion, or after ischemia at the time of restoration of blood flow to the kidney.

We included five experimental groups to investigate whether RIC could protect the kidney from ischemic injury. Indeed, the IRI group demonstrated significantly more serologic and histological evidence of renal damage than the sham group, verifying the induction of ischemia/ reperfusion injury. Additionally, $\mathrm{SCr}$ and BUN levels in the RIPerC, RIPostC and RIPerC + RIPostC groups were significantly lower than those in the IRI group $(P<0.05)$, demonstrating the protective effect of RIC. There was no significant difference in $\mathrm{SCr}$ or BUN levels between the three RIC groups $(P>0.05)$.

MPO accounts for about $5 \%$ of the dry cell weight of neutrophils. The level of MPO activity in renal tissue represents the quantitative expression of neutrophil activity and infiltration into the kidneys. MPO dose not induce cell apoptosis, but in 1966, Yang et al. demonstrated that the release of proteinase 3 (PR3) and elastase by activated neutrophils during acute inflammation, may result in vascular damage by causing endothelial cell apoptosis [10, 11]. In other words, MPO activity may indirectly reflect the extent of renal tubular epithelial cell interstitial cellular infiltration; c, d, e RIPerC, RIPostC, and RIPerC + RIPostC treatment (RIPerC, RIPostC, and RIPerC + RIPostC groups): the degree of renal graft injury clearly less severe than that in the IRI group (B)

apoptosis [12]. The IRI, RIPerC, RIPostC, and RIPerC + RIPostC groups all showed significantly higher MPO activity than the sham group $(P<0.05)$. This indicates that after ischemia-reperfusion, a large number of neutrophils are activated, and these infiltrate into the local ischemic tissues. MPO activities in the RIPerC, RIPostC, and RIPerC + RIPostC groups were lower than those in the IRI group $(P<0.05)$, once again supporting the protective effect of RIC. The degree of nephritic tubular injury in the RIPerC, RIPostC, and RIPerC + RIPostC groups was less prominent than that seen in the IRI group $(P<0.05)$. These results suggest that the RIPerC, RIPostC, and RIPerC + RIPostC groups experienced less renal tubular damage through a reduction of neutrophil accumulation and renal tubular epithelial cell apoptosis in ischemic renal ischemic tissue.

MDA is the main metabolite of lipid peroxidation within the body, and elevated levels indirectly reflect a higher degree of cell damage in the body. SOD plays a key role in the body's oxidation and antioxidative balance; this enzyme protects cells from damage by scavenging the superoxide anion radical (O2-). SOD activity indirectly reflects the level of free radical scavenging activity within the body, and the level of MDA indirectly reflects the severity of cellular injury from free radical attack. The IRI, RIPerC, RIPostC, and RIPerC + RIPostC groups all demonstrated higher 
renal tissue MDA content than the sham surgery group. SOD activity was significantly lower in the IRI group than SOD activities in the RIPerC, RIPostC, and RIPerC + RIPostC treatment groups $(P<0.05)$. The RIPerC, RIPostC, and RIPerC + RIPostC groups were able to maintain a higher SOD activity than the IRI group. This enables the destruction of more oxygen-free radicals, and reduces oxygen-free radical-mediated lipid peroxidation. The higher SOD activity in the RIC groups leads to a reduction in the degree of renal IRI and may account for a significant portion of RIC's protective effect.

Our results indicated that RIC reduced the intensity of renal inflammation intensity, protecting the kidneys from IRI to some extent. There was no significant difference between the three RIC treatment groups. These three different approaches likely function via the same mechanism of action to reduce renal IRI. In clinical studies, the protective role of RIC in preventing IRI has been controversial. Ravlo et al. [13], Wu et al. [14] and Søndergaard et al. [8] observed that RIC can protect kidney transplant patients from renal IRI. Huang et al. also observed similar protective effects in kidney resection, but Chen et al. [15] found no improvement in renal function when RIC was used in living donor kidney transplantation. Further studies are necessary to determine the optimal techniques and indications for RIC in the future, especially with regard to clinical trials.

\section{Conclusion}

We have demonstrated the protective effects of RIC in limiting IRI in the rat kidney. RIPerC, RIPostC, and RIPerC + RIPostC treatments were all equally beneficial, and possibly exerted their effects by enhancing antioxidant activity and decreasing inflammation in the injured renal tissues. The RIPerC + RIPostC protocol did not show an additive effect from the combination of perconditioning and postconditioning. This may be because each protocol consisted of 4 remote $\mathrm{I} / \mathrm{R}$ cycles, which in the RIPerC + RIPostC protocol were split evenly between perconditioning and postconditioning.

\begin{abstract}
Acknowledgments We certify that there is no actual or potential conflict of interest in relation to this article. Haibo Jiang and Xiaoqing Sun conceived and designed the study. Renfu Chen, Song Xue, Haitao Zhu, and Xiaolei Sun analyzed and interpreted the data. Xiaolei Sun collected the data. Haibo Jiang wrote the manuscript. Xiaoqing Sun and Renfu Chen critically revised the manuscript. Xiaoqing Sun approved the final version of the manuscript.
\end{abstract}

Open Access This article is distributed under the terms of the Creative Commons Attribution License which permits any use, distribution, and reproduction in any medium, provided the original author(s) and the source are credited.

\section{References}

1. Johnson KJ, Weinberg JM (1993) Postischemic renal injury due to oxygen radicals. Curr Opin Nephrol Hypertens 2(4):625-635

2. Eltzschig HK, Eckle T (2011) Ischemia and reperfusion-from mechanism to translation. Nat Med 17(11):1391-1401. doi:10. 1038/nm.2507

3. Kadkhodaee M, Seifi B, Najafi A, Sedaghat Z (2011) First report of the protective effects of remote per- and postconditioning on ischemia/reperfusion-induced renal injury. Transplantation 92(10):e55. doi:10.1097/TP.0b013e31823411f8

4. Schmidt MR, Smerup M, Konstantinov IE, Shimizu M, Li J, Cheung $M$ et al (2007) Intermittent peripheral tissue ischemia during coronary ischemia reduces myocardial infarction through a KATP-dependent mechanism: first demonstration of remote ischemic perconditioning. Am J Physiol Heart Circ Physiol 292(4):H1883-H1890. doi:10.1152/ajpheart.00617.2006

5. Kerendi F, Kin H, Halkos ME, Jiang R, Zatta AJ, Zhao ZQ et al (2005) Remote postconditioning. Brief renal ischemia and reperfusion applied before coronary artery reperfusion reduces myocardial infarct size via endogenous activation of adenosine receptors. Basic Res Cardiol 100(5):404-412. doi:10.1007/ s00395-005-0539-2

6. Wever KE, Warle MC, Wagener FA, van der Hoorn JW, Masereeuw R, van der Vliet JA et al (2011) Remote ischaemic preconditioning by brief hind limb ischaemia protects against renal ischaemia-reperfusion injury: the role of adenosine. Nephrol Dial Transplant 26(10):3108-3117. doi:10.1093/ndt/gfr103

7. Saxena P, Newman MA, Shehatha JS, Redington AN, Konstantinov IE (2010) Remote ischemic conditioning: evolution of the concept, mechanisms, and clinical application. J Card Surg 25(1):127-134. doi:10.1111/j.1540-8191.2009.00820.x

8. Soendergaard P, Krogstrup NV, Secher NG, Ravlo K, Keller AK, Toennesen E et al (2012) Improved GFR and renal plasma perfusion following remote ischaemic conditioning in a porcine kidney transplantation model. Transpl Int 25(9):1002-1012. doi:10.1111/j.1432-2277.2012.01522.x

9. Huang J, Chen Y, Dong B, Kong W, Zhang J, Xue W et al (2013) Effect of remote ischaemic preconditioning on renal protection in patients undergoing laparoscopic partial nephrectomy: a 'blinded' randomised controlled trial. BJU Int 112(1):74-80. doi:10.1111/ bju. 12004

10. Yang JJ, Kettritz R, Falk RJ, Jennette JC, Gaido ML (1996) Apoptosis of endothelial cells induced by the neutrophil serine proteases proteinase 3 and elastase. Am J Pathol 149(5): $1617-1626$

11. Taekema-Roelvink ME, van Kooten C, Janssens MC, Heemskerk E, Daha MR (1998) Effect of anti-neutrophil cytoplasmic antibodies on proteinase 3-induced apoptosis of human endothelial cells. Scand J Immunol 48(1):37-43

12. Koshio O, Nagao T, Mabuchi A, Ono Y, Suzuki K (2012) Apoptotic signaling in endothelial cells with neutrophil activation. Mol Cell Biochem 363(1-2):269-280. doi:10.1007/s11010011-1179-5

13. Ravlo K, Koefoed-Nielsen P, Secher N, Sondergaard P, Keller AK, Petersen MS et al (2012) Effect of remote ischemic conditioning on dendritic cell number in blood after renal transplantation: flow cytometry in a porcine model. Transpl Immunol 26(2-3):146-150. doi:10.1016/j.trim.2011.10.006

14. Wu J, Feng X, Huang H, Shou Z, Zhang X, Wang R et al (2014) Remote ischemic conditioning enhanced the early recovery of renal function in recipients after kidney transplantation: a randomized controlled trial. J Surg Res 188(1):303-308. doi:10. $1016 / j . j s s .2013 .06 .058$ 
15. Chen Y, Zheng H, Wang X, Zhou Z, Luo A, Tian Y (2013)

Remote ischemic preconditioning fails to improve early renal function of patients undergoing living-donor renal transplantation: a randomized controlled trial. Transplantation 95(2):e4-e6. doi:10.1097/TP.0b013e3182782f3a 